\title{
RATIONAL DIOPHANTINE SEXTUPLES CONTAINING TWO REGULAR QUADRUPLES AND ONE REGULAR QUINTUPLE
}

\author{
ANDREJ DUJELLA, MATIJA KAZALICKI, AND VINKO PETRIČEVIĆ \\ Abstract. A set of $m$ distinct nonzero rationals $\left\{a_{1}, a_{2}, \ldots, a_{m}\right\}$ such that \\ $a_{i} a_{j}+1$ is a perfect square for all $1 \leq i<j \leq m$, is called a rational Dio- \\ phantine $m$-tuple. It is proved recently that there are infinitely many rational \\ Diophantine sextuples. In this paper, we construct infinite families of rational \\ Diophantine sextuples with special structure, namely the sextuples containing \\ quadruples and quintuples of certain type.
}

\section{INTRODUCTION}

A Diophantine $m$-tuple is a set of $m$ distinct positive integers with the property that the product of any two of its distinct elements plus 1 is a square. Fermat found the first Diophantine quadruple in integers $\{1,3,8,120\}$. If a set of $m$ nonzero rationals has the same property, then it is called a rational Diophantine $m$-tuple. The first example of a rational Diophantine quadruple was the set

$$
\left\{\frac{1}{16}, \frac{33}{16}, \frac{17}{4}, \frac{105}{16}\right\}
$$

found by Diophantus. Euler proved that the exist infinitely many rational Diophantine quintuples (see [16]), in particular he was able to extend the integer Diophantine quadruple found by Fermat, to the rational quintuple

$$
\left\{1,3,8,120, \frac{777480}{8288641}\right\} \text {. }
$$

Stoll [19] recently showed that this extension is unique. Therefore, the Fermat set $\{1,3,8,120\}$ cannot be extended to a rational Diophantine sextuple.

In 1969, using linear forms in logarithms of algebraic numbers and a reduction method based on continued fractions, Baker and Davenport [1 proved that if $d$ is a positive integer such that $\{1,3,8, d\}$ forms a Diophantine quadruple, then $d$ has to be 120 . This result motivated the conjecture that there does not exist a Diophantine quintuples in integers. The conjecture has been proved recently by He, Togbé and Ziegler [15] (see also [2, 6]).

In the other hand, it is not known how large can be a rational Diophantine tuple. In 1999, Gibbs found the first example of rational Diophantine sextuple [12]

$$
\left\{\frac{11}{192}, \frac{35}{192}, \frac{155}{27}, \frac{512}{27}, \frac{1235}{48}, \frac{180873}{16}\right\} \text {. }
$$

In 2017 Dujella, Kazalicki, Mikić and Szikszai 10. proved that there are infinitely many rational Diophantine sextuples, while Dujella and Kazalicki [9] (inspired by the work of Piezas [18]) described another construction of parametric families of rational Diophantine sextuples. Recently, Dujella, Kazalicki and Petričević [11] proved that there are infinitely many rational Diophantine sextuples such that

2010 Mathematics Subject Classification. Primary 11D09; Secondary 11G05.

Key words and phrases. rational Diophantine sextuples, regular Diophantine quadruples, regular Diophantine quintuples, elliptic curves. 
denominators of all the elements (in the lowest terms) in the sextuples are perfect squares. No example of a rational Diophantine septuple is known. The Lang conjecture on varieties of general type implies that the number of elements of a rational Diophantine tuple is bounded by an absolute constant (see Introduction of [10). For more information on Diophantine $m$-tuples see the survey article 8 .

Although the constructions of infinitely families of rational Diophantine sextuples in [9] and 10] are essentially different, they have one common feature. Namely, in both constructions (and also in [11, which is a special case of [9]) the sextuples contain two regular rational Diophantine quintuples. The quintuple $\{a, b, c, d, e\}$ is called regular if

$$
(a b c d e+2 a b c+a+b+c-d-e)^{2}=4(a b+1)(a c+1)(b c+1)(d e+1)
$$

(see [3, 5, 7, 13]). Similarly, the quadruple $\{a, b, c, d\}$ is called regular if

$$
(a+b-c-d)^{2}=4(a b+1)(c d+1)
$$

(see [5] for characterization of regular Diophantine quadruples and quintuples in terms of corresponding elliptic curves).

In [14, Gibbs collected over 1000 examples of rational Diophantine sextuples with relatively small numerators and denominators. These examples are also sorted in [14] according to their structure, which includes information of regular quadruples and quintuples which they contain. We have extended the search for sextuples with small height and included also examples with mixed signs (in [14] only sextuples with positive elements were considered). We have observed a significant number of sextuples which contain exactly one regular Diophantine quintuple and two regular Diophantine quadruples. Thus, in this paper we study rational Diophantine sextuples having this structure. Our main result is the following theorem.

Theorem 1. There are infinitely many rational Diophantine sextuples which contain one regular Diophantine quintuple and two regular Diophantine quadruples.

\section{Parametrizations of Diophantine triples}

Let $\left\{a_{1}, a_{2}, a_{3}\right\}$ be a rational Diophantine triple and let $a_{2}=\left(r^{2}-1\right) / a_{1}$ and $a_{3}=\left(s^{2}-1\right) / a_{1}$ for rationals $r$ and $s$. By putting $a_{2} a_{3}+1=\left(a_{2} s^{2}-a_{2}+a_{1}\right) / a_{1}=$ $(1+(s-1) t)^{2}$, we get

$$
a_{3}=\frac{-4 t(t-1)\left(a_{1} t-a_{2}\right)}{\left(-a_{2}+a_{1} t^{2}\right)^{2}} .
$$

This parametrization of Diophantine triples was used in [7] in construction of certain rational Diophantine sextuples. Here we will use an equivalent, but simpler and more aesthetic parametrization due to Lasić [17, which is symmetric in the three involved parameters:

$$
\begin{aligned}
& a_{1}=\frac{2 t_{1}\left(1+t_{1} t_{2}\left(1+t_{2} t_{3}\right)\right)}{\left(-1+t_{1} t_{2} t_{3}\right)\left(1+t_{1} t_{2} t_{3}\right)}, \\
& a_{2}=\frac{2 t_{2}\left(1+t_{2} t_{3}\left(1+t_{3} t_{1}\right)\right)}{\left(-1+t_{1} t_{2} t_{3}\right)\left(1+t_{1} t_{2} t_{3}\right)}, \\
& a_{3}=\frac{2 t_{3}\left(1+t_{3} t_{1}\left(1+t_{1} t_{2}\right)\right)}{\left(-1+t_{1} t_{2} t_{3}\right)\left(1+t_{1} t_{2} t_{3}\right)} .
\end{aligned}
$$

The connection between two parametizations is given by

$$
\begin{gathered}
t_{1}=\frac{a_{1}}{r-1}, \quad t_{2}=\frac{-\left(1-r^{2}+a_{1}^{2} t^{2}\right)}{2(t-1) a_{1}}, \quad t_{3}=\frac{2 a_{1} t(t-1)}{1-r^{2}+a_{1}^{2} t^{2}}, \\
a_{1}=\frac{2 t_{1}\left(1+t_{1} t_{2}\left(1+t_{2} t_{3}\right)\right)}{\left(-1+t_{1} t_{2} t_{3}\right)\left(1+t_{1} t_{2} t_{3}\right)}, \quad r=\frac{1+2 t_{1} t_{2}+2 t_{1} t_{2}^{2} t_{3}+t_{2}^{2} t_{3}^{2} t_{1}^{2}}{\left(-1+t_{1} t_{2} t_{3}\right)\left(1+t_{1} t_{2} t_{3}\right)}, \quad t=-t_{2} t_{3} .
\end{gathered}
$$




\section{New COnstruction of families of Diophantine SeXtuples}

Let $\left\{a_{1}, a_{2}, a_{3}, a_{4}\right\}$ and $\left\{a_{1}, a_{2}, a_{3}, a_{5}\right\}$ be regular Diophantine quadruples, i.e. $a_{4}$ and $a_{5}$ are solutions of the quadratic equation

$$
\left(a_{1}+a_{2}-a_{3}-x\right)^{2}-4\left(a_{1} a_{2}+1\right)\left(a_{3} x+1\right)=0 .
$$

We obtain that

$$
\begin{aligned}
& a_{4}=\frac{-2\left(1-t_{3}+t_{2} t_{3}\right)\left(t_{3} t_{1}+1-t_{1}\right)\left(-t_{2}+1+t_{1} t_{2}\right)\left(-1+t_{1} t_{2} t_{3}\right)}{\left(1+t_{1} t_{2} t_{3}\right)^{3}}, \\
& a_{5}=\frac{2\left(t_{3}+t_{2} t_{3}+1\right)\left(t_{3} t_{1}+t_{1}+1\right)\left(1+t_{2}+t_{1} t_{2}\right)\left(1+t_{1} t_{2} t_{3}\right)}{\left(-1+t_{1} t_{2} t_{3}\right)^{3}} .
\end{aligned}
$$

In order that $\left\{a_{1}, a_{2}, a_{3}, a_{4}, a_{5}\right\}$ be a rational Diophantine quintuple, it remains to satisfy the condition that $a_{4} a_{5}+1$ is a perfect square. We obtain the condition that

$$
\begin{aligned}
p\left(t_{1}, t_{2}, t_{3}\right)= & \left(-8 t_{2}^{3} t_{3}^{3}-8 t_{3}^{2} t_{2}^{2}-3 t_{3}^{4} t_{2}^{4}+4 t_{2}^{2}+4 t_{2}^{2} t_{3}^{4}+4 t_{2}^{4} t_{3}^{2}+8 t_{2}^{3} t_{3}\right) t_{1}^{4} \\
+ & \left(8 t_{2}^{2} t_{3}-16 t_{2} t_{3}^{2}-8 t_{2}^{3} t_{3}^{2}+8 t_{2}-8 t_{2}^{3} t_{3}^{4}-8 t_{2}^{4} t_{3}^{3}-8 t_{2}^{2} t_{3}^{3}+8 t_{3}^{4} t_{2}\right) t_{1}^{3} \\
+ & \left(-8 t_{3}^{2}-8 t_{2}^{2}-8 t_{2} t_{3}-8 t_{2}^{3} t_{3}^{3}-8 t_{2}^{2} t_{3}^{4}+8 t_{3}^{3} t_{2}+4 t_{3}^{4}+4-18 t_{3}^{2} t_{2}^{2}\right. \\
& \left.+4 t_{3}^{4} t_{2}^{4}-8 t_{2}^{4} t_{3}^{2}-16 t_{2}^{3} t_{3}\right) t_{1}^{2} \\
+ & \left(8 t_{2}^{4} t_{3}^{3}-8 t_{2}^{2} t_{3}-16 t_{2}^{2} t_{3}^{3}-8 t_{2} t_{3}^{2}-8 t_{2}+8 t_{3}^{3}+8 t_{2}^{3} t_{3}^{2}-8 t_{3}\right) t_{1} \\
& -3-8 t_{2} t_{3}+4 t_{2}^{4} t_{3}^{2}-8 t_{3}^{2} t_{2}^{2}+4 t_{3}^{2}+4 t_{2}^{2}+8 t_{2}^{3} t_{3}
\end{aligned}
$$

is a perfect square. We compute the discriminant of $p\left(t_{1}, t_{2}, t_{3}\right)$ with the respect to $t_{1}$ and factorize it. One of the factors is

$$
p_{1}\left(t_{2}, t_{3}\right)=3+10 t_{2} t_{3}-3 t_{3}^{2}+3 t_{3}^{2} t_{2}^{2} .
$$

The condition $p_{1}\left(t_{2}, t_{3}\right)=0$ leads to $9 t_{3}^{2}+16$ be a perfect square, say $9 t_{3}^{2}+16=$ $\left(3 t_{3}+u\right)^{2}$. We get

$$
\begin{aligned}
& t_{3}=\frac{16-u^{2}}{6 u}, \\
& t_{2}=\frac{u^{2}+10 u+16}{(u-4)(u+4)} .
\end{aligned}
$$

Inserting this in (1), we obtain that $a_{4} a_{5}+1$ is a perfect square. Thus, we obtained a two-parametric family (in parameters $t_{1}$ and $u$ ) of rational Diophantine quintuples which contain two regular quadruples (let us mention that a one-parametric family of rational Diophantine quintuples with this property was constructed in [4]).

Now we extend the nonregular quadruple $\left\{a_{1}, a_{3}, a_{4}, a_{5}\right\}$ to regular quintuples $\left\{a_{1}, a_{3}, a_{4}, a_{5}, a_{6}\right\}$ and $\left\{a_{1}, a_{3}, a_{4}, a_{5}, a_{7}\right\}$, i.e. $a_{6}$ and $a_{7}$ are solutions of the quadratic equation

$\left(a_{1} a_{3} a_{4} a_{5} x+2 a_{1} a_{3} a_{4}+a_{1}+a_{3}+a_{4}-a_{5}-x\right)^{2}-4\left(a_{1} a_{3}+1\right)\left(a_{1} a_{4}+1\right)\left(a_{3} a_{4}+1\right)\left(a_{5} x+1\right)=0$.

We will not use $a_{7}$ is our construction, so we give here only the value of $a_{6}$ :

$$
\begin{aligned}
a_{6} & =6(u+4)(u+8)(u+2)(u-4)\left(2 t_{1} u^{2}+3 u^{2}+20 t_{1} u+12 u+32 t_{1}\right) \\
& \times\left(t_{1} u^{2}+10 t_{1} u+16 t_{1}-6 u\right)\left(t_{1} u^{2}+10 t_{1} u+16 t_{1}+6 u\right)\left(t_{1} u^{2}+10 t_{1} u+16 t_{1}-24-6 u\right) \\
& \times\left(4096 t_{1}^{2}+15360 t_{1}^{2} u+15168 t_{1}^{2} u^{2}+5920 t_{1}^{2} u^{3}+948 t_{1}^{2} u^{4}+60 t_{1}^{2} u^{5}+t_{1}^{2} u^{6}-12288 t_{1} u\right. \\
& \left.-7680 t_{1} u^{2}+480 t_{1} u^{4}+48 t_{1} u^{5}-5184 u^{2}-2592 u^{3}-324 u^{4}\right)^{-2} .
\end{aligned}
$$

The only missing condition in order that $\left\{a_{1}, a_{2}, a_{3}, a_{4}, a_{5}, a_{6}\right\}$ be a rational Diophantine sextuple is that $a_{2} a_{6}+1$ is a perfect square. This condition leads to the quartic in $t_{1}$ over $\mathbb{Q}(u)$ : 


$$
\begin{aligned}
& \left(u^{12}+120 u^{11}+5496 u^{10}+125600 u^{9}+1639440 u^{8}+13075200 u^{7}+65656320 u^{6}\right. \\
& \left.\quad+209203200 u^{5}+419696640 u^{4}+514457600 u^{3}+360185856 u^{2}+125829120 u+16777216\right) t_{1}^{4} \\
& +\left(24 u^{12}++1296 u^{11}+32256 u^{10}+446208 u^{9}+3461760 u^{8}+13047552 u^{7}-208760832 u^{5}\right. \\
& \left.\quad-886210560 u^{4}-1827667968 u^{3}-2113929216 u^{2}-1358954496 u-402653184\right) t_{1}^{3} \\
& +\left(36 u^{12}+1296 u^{11}+18072 u^{10}+48096 u^{9}-1681632 u^{8}-22516992 u^{7}-127051776 u^{6}\right. \\
& \left.\quad-360271872 u^{5}-430497792 u^{4}+197001216 u^{3}+1184366592 u^{2}+1358954496 u+603979776\right) t_{1}^{2} \\
& +\left(-432 u^{11}-15552 u^{10}-259200 u^{9}-2267136 u^{8}-9116928 u^{7}+145870848 u^{5}\right. \\
& \left.+580386816 u^{4}+1061683200 u^{3}+1019215872 u^{2}+452984832 u\right) t_{1} \\
& +1296 u^{10}+41472 u^{9}+31643136 u^{6}+670032 u^{8}+6054912 u^{7}+96878592 u^{5}+171528192 u^{4} \\
& +169869312 u^{3}+84934656 u^{2}=z^{2} .
\end{aligned}
$$

Since this quartic has a $\mathbb{Q}(u)$-rational point at infinity, it can be transformed by birational transformations into an elliptic curve over $\mathbb{Q}(u)$ (the singular point at infinity on the quartic corresponds to the point at infinity and an additional point $P_{1}$ on the elliptic curve). The quartic have another $\mathbb{Q}(u)$-rational point corresponding to $t_{1}=\frac{-3(u+4) u}{2\left(u^{2}+10 u+16\right)}$. It gives $a_{6}=0$, so it does not yield a rational Diophantine sextuples. However, if we denote the corresponding point on the elliptic curve by $P_{2}$, then the point $2 P_{2}$ on the elliptic curve corresponds to the point with

$$
t_{1}=\frac{3\left(3 u^{4}+40 u^{3}+368 u^{2}+1280 u+1024\right)}{4\left(u^{2}+10 u+16\right)(u+20) u}
$$

on the quartic, and by inserting this value, we obtain the parametric family of rational Diophantine sextuples

$$
\begin{aligned}
& \left\{\frac{-12 u(u+4)\left(3 u^{4}+8 u^{3}+224 u^{2}+576 u+512\right)\left(3 u^{3}+28 u^{2}+256 u+256\right)}{(u+8)(u+2)(u-4)\left(3 u^{3}+8 u^{2}+144 u+128\right)\left(3 u^{4}+48 u^{3}+528 u^{2}+1280 u+1024\right)},\right. \\
& \frac{8 u(u+20)\left(3 u^{5}+8 u^{4}+64 u^{3}-640 u^{2}-2304 u-2048\right)(u+8)(u+2)}{3(u+4)(u-4)\left(3 u^{3}+8 u^{2}+144 u+128\right)\left(3 u^{4}+48 u^{3}+528 u^{2}+1280 u+1024\right)}, \\
& \underline{2(u+4)(u-4)\left(39 u^{7}+776 u^{6}+8096 u^{5}+48640 u^{4}+226048 u^{3}+587776 u^{2}+770048 u+393216\right)} \\
& 3(u+8)(u+2)\left(3 u^{3}+8 u^{2}+144 u+128\right)\left(3 u^{4}+48 u^{3}+528 u^{2}+1280 u+1024\right) \\
& \frac{-8\left(u^{2}+4 u+32\right)\left(3 u^{3}+14 u^{2}-40 u-64\right)\left(9 u^{3}+8 u^{2}+112 u+384\right)\left(3 u^{4}+48 u^{3}+528 u^{2}+1280 u+1024\right)}{\text {, }} \\
& 3(u+8)(u+4)(u+2)(u-4)\left(3 u^{3}+8 u^{2}+144 u+128\right)^{3} \\
& \frac{4 u(u+2)\left(17 u^{2}+48 u+48\right)\left(3 u^{5}+8 u^{4}-176 u^{3}-2944 u^{2}-9216 u-8192\right)\left(3 u^{3}+8 u^{2}+144 u+128\right)(u+8)^{2}}{3(u+4)(u-4)\left(3 u^{4}+48 u^{3}+528 u^{2}+1280 u+1024\right)^{3}} \\
& \left.\frac{12(u+2)(u-4)(5 u+8)(u+4)\left(3 u^{2}+8 u+64\right)\left(3 u^{3}+8 u^{2}+144 u+128\right)\left(3 u^{4}+48 u^{3}+528 u^{2}+1280 u+1024\right)}{(u+8)\left(16384+69632 u+64768 u^{2}+22272 u^{3}+3680 u^{4}+576 u^{5}+9 u^{6}\right)^{2}}\right\}
\end{aligned}
$$

which satisfies the properties from Theorem 1 .

E.g. for $u=-1$ we get the rational Diophantine sextuple

$$
\left\{\frac{27900}{17479}, \frac{471352}{112365}, \frac{261770}{17479}, \frac{185535272}{419265}, \frac{63737828}{526368735}, \frac{79554420}{408480247}\right\} \text {. }
$$

By taking other linear combinations of the points $P_{1}$ and $P_{2}$ we can also obtain (more complicated) families of rational Diophantine sextuples.

Acknowledgements. The authors were supported by the Croatian Science Foundation under the project no. IP-2018-01-1313. The authors acknowledge support from the QuantiXLie Center of Excellence, a project co-financed by the Croatian Government and European Union through the European Regional Development Fund - the Competitiveness and Cohesion Operational Programme (Grant KK.01.1.1.01.0004). The authors acknowledge the usage of the supercomputing resources of Division of Theoretical Physics at Ruđer Bošković Institute, as well 
as the computing resources at Department of Mathematics, University of Zagreb which were provided by Croatian Science Foundation grant HRZZ-9345

\section{REFERENCES}

[1] A. Baker and H. Davenport, The equations $3 x^{2}-2=y^{2}$ and $8 x^{2}-7=z^{2}$, Quart. J. Math. Oxford Ser. (2) 20 (1969), 129-137.

[2] M. Bliznac Trebješanin and A. Filipin, Nonexistence of D(4)-quintuples, J. Number Theory 194 (2019), 170-217.

[3] A. Dujella, On Diophantine quintuples, Acta Arith. 81 (1997), 69-79.

[4] A. Dujella, Diophantine triples and construction of high-rank elliptic curves over $\mathbb{Q}$ with three non-trivial 2-torsion points, Rocky Mountain J. Math. 30 (2000), 157-164.

[5] A. Dujella, Irregular Diophantine m-tuples and elliptic curves of high rank, Proc. Japan Acad. Ser. A Math. Sci. 76 (2000), 66-67.

[6] A. Dujella, There are only finitely many Diophantine quintuples, J. Reine Angew. Math. 566 (2004), 183-214.

[7] A. Dujella, Rational Diophantine sextuples with mixed signs, Proc. Japan Acad. Ser. A Math. Sci. 85 (2009), 27-30.

[8] A. Dujella, What is ... a Diophantine m-tuple?, Notices Amer. Math. Soc. 63 (2016), 772774 .

[9] A. Dujella and M. Kazalicki, More on Diophantine sextuples, in: Number Theory - Diophantine problems, uniform distribution and applications, Festschrift in honour of Robert F. Tichy's 60th birthday (C. Elsholtz, P. Grabner, Eds.), Springer-Verlag, Berlin, 2017, pp. $227-235$.

[10] A. Dujella, M. Kazalicki, M. Mikić and M. Szikszai, There are infinitely many rational Diophantine sextuples, Int. Math. Res. Not. IMRN 2017 (2) (2017), 490-508.

[11] A. Dujella, M. Kazalicki and V. Petričević, There are infinitely many rational Diophantine sextuples with square denominators, preprint, 2019.

[12] P. Gibbs, Some rational Diophantine sextuples, Glas. Mat. Ser. III 41 (2006), 195-203.

[13] P. Gibbs, Regular rational Diophantine sextuples, preprint, 2016.

[14] P. Gibbs, A survey of rational diophantine sextuples of low height, preprint, 2016.

[15] B. He, A. Togbé and V. Ziegler, There is no Diophantine quintuple, Trans. Amer. Math. Soc., to appear.

[16] T. L. Heath, Diophantus of Alexandria. A Study in the History of Greek Algebra. Powell's Bookstore, Chicago; Martino Publishing, Mansfield Center, 2003.

[17] L. Lasić, personal communication, 2017.

[18] T. Piezas, Extending rational Diophantine triples to sextuples, http://mathoverflow.net/questions/233538/extending-rational-diophantine-triples-to-sextuples

[19] M. Stoll, Diagonal genus 5 curves, elliptic curves over $\mathbb{Q}(t)$, and rational diophantine quintuples, Acta Arith., to appear.

Department of Mathematics, Faculty of Science, University of Zagreb, BijeničKa Cesta 30, 10000 ZaGreb, Croatia

E-mail address, A. Dujella: duje@math.hr

Department of Mathematics, Faculty of Science, University of Zagreb, Bijenička Cesta 30, 10000 Zagreb, Croatia

E-mail address, M. Kazalicki: matija.kazalicki@math.hr

Department of Mathematics, Faculty of Science, University of Zagreb, Bijenička CEsta 30, 10000 Zagreb, Croatia

E-mail address, V. Petričević: vpetrice@math.hr 